\title{
CAPACIDADES DIRECTIVAS Y ESTRATEGIAS GENÉRICAS EN LA DOP CAVA
}

\author{
Juan Ramón Ferrera (*) \\ ${ }^{a}$ Universidad Politécnica de Madrid (Madrid, juanramon.ferrer@upm.es)

\section{Resumen}

La denominación de origen Cava es el mayor exportador de la industria vitivinícola española. Sin embargo, en los últimos veinte años el crecimiento de las exportaciones ha sido menor que el de otras denominaciones de origen, y también menor su evolución en el mercado interior. El aumento del entorno competitivo global obliga a todas las bodegas a conseguir la ventaja competitiva, y convierte en fundamental conocer cuáles son las características de las bodegas que alcanzan un mejor desempeño. Este trabajo aborda el análisis de la ventaja competitiva a través de dos acercamientos, los recursos y capacidades, y la estrategia. En los recursos se estudian las capacidades directivas y en la estrategia el modelo de Robinson y Pearce. El objetivo es doble, por un lado, conocer el nivel de las capacidades de gestión en las bodegas de Cava con denominación de origen protegida, y por otro lado conocer la influencia de las capacidades de gestión, y las estrategias genéricas en el rendimiento empresarial. Se analiza un conjunto de 66 bodegas mediante la prueba de U Mann Whitney, y se utiliza la técnica de la regresión bayesiana para determinar la relación entre las variables.

Palabras clave: ventaja competitiva, sector del vino, desempeño empresarial, recursos y capacidades.

\section{Introducción}

La DOP Cava es el mayor exportador de vino embotellado de España, ligeramente por delante de la DOP Rioja, representando alrededor del $27 \%$ del volumen total de exportación de vino nacional (MAPA, 2020). La historia del Cava comienza con la epidemia de filoxera que asoló los viñedos franceses en 1870, lo que supuso una oportunidad para que toda la industria vitivinícola española se convirtiera en proveedor del país vecino (Fernández \& Pinilla, 2014). También fue el inicio de la investigación de Josep Raventos con el método tradicional del champagne, con uvas autóctonas de Cataluña, en especial las variedades parellada, macabeo y xarel-lo. Cuando la filoxera destruyó los viñedos del Penedès a principios del siglo XX, la mayor parte de los agricultores aprovecharon para sustituir las tradicionales uvas tintas por las variedades con las que Josep Raventos experimentó para hacer cava (Saito \& Takenaka, 2004). En 1972 se crea el Consejo Regulador de los Vinos Espumosos y se aprueba la denominación "Cava" para designar el vino espumoso español (CRC, 2021a).

Una característica única de la DOP Cava, que no comparten otras DOP, es que las bodegas pertenecientes a la DOP Cava que elaboran vino espumoso, pueden ubicarse fuera de la zona de producción original del Penedès, aunque el 90\% de las bodegas con DOP de Cava se sitúan en el Penedès (Saito y Takenaka, 2004).

La superficie dedicada al Cava fue de 32.009 hectáreas en la campaña 2000-01, frente a 57.636 hectáreas en Rioja y 673.626 hectáreas en todas las DOP de España. Los datos de la campaña 2018-19 reflejan una superficie de 37.955 hectáreas para Cava, 66.239 hectáreas en Rioja y 648.631 hectáreas en el total nacional. A lo largo de este período de 19 años, podemos observar, por tanto, un crecimiento del Cava del $18 \%$, un crecimiento del Rioja del 15\% y una disminución global de las DOP totales del 3,7\% (MAPA, 2020). El valor generado por hectárea cultivada y por bodega en el periodo 2016-17 a 2018-19 es mayor en Cava que en otras DOPs. Así el valor generado por ha en Cava es de alrededor de 20.000 euros/ha, seguida de Rioja con alrededor de 15.000 y la media nacional situada en algo más de 6.000 euros/ha (MAPA, 2020). Mientras que el valor generado por bodega en el periodo 2016-17 a 2018-19, se sitúa muy cerca de los 2 millones de euros por bodega en Cava, alrededor de 1,2 millones en Rioja y por debajo de 0,9 millones de euros por bodega en la media nacional

\section{Objetivo}

El objetivo de esta investigación es doble, por una parte, conocer cuál es la posición de DOP Cava con respecto a Rioja en el recursos capacidades directivas, y por otra determinar si las capacidades directivas y qué tipologías estratégicas son las que están relacionadas con el mejor desempeño empresarial en la DOP Cava.

\section{Metodología}

La relación de empresas que operan en el sector vitivinícola en las DOP Cava y Rioja se ha elaborado a partir de dos fuentes de información. La primera fuente es la base de datos del Sistema Ibérico de Análisis del Balance (SABI, 2017), tomando aquellas empresas que se encuentran registradas y en activo, en el epígrafe 11.02 de la Clasificación Nacional de Actividades Económicas (CNAE) correspondiente a 
“Empresas Vitícolas". La segunda fuente es el registro público de empresas vitivinícolas que existen en las distintas regiones de Denominación de Origen Protegida (DOP).

Se envió una encuesta por correo electrónico a los gerentes, personal de marketing o directores de producción con recordatorios telefónicos posteriores. Al final del proceso se recibieron un total de 66 respuestas válidas, 20 en Cava y 46 en Rioja, lo que representa el 13\% del total de la muestra; considerado una tasa de respuesta válida para los sectores industriales según Baruch \& Holtom (2008). La tipología de las bodegas de Cava y Rioja, presentan rasgos similares en cuanto a su tamaño y capacidad, tomando como referencia el número de empleados, así Cava presenta una media de 10,75 empleados, mientras que en Rioja este dato es de 10,41, no considerándose la existencia de sesgo entre los grupos, T test, no significativo (sig $=0,961)$.

Para determinar las diferencias existentes en las capacidades de gestión entre las DOPs Cava y Rioja, se utiliza la prueba U de Mann-Whitney para dos muestras independientes.

Para determinar la relación entre las estrategias, las capacidades directivas y el performance financiero se desarrolla regresión bayesiana, por su mayor fiabilidad cuando el número de casos es bajo y cuando no se puede asegurar la normalidad de las variables (Block et al., 2011). El modelo de análisis propuesto es el siguiente:

$$
Y_{j}=\beta_{0}+\beta_{I} S_{j}+\beta_{2} C D_{j}+\beta_{3} A_{j}+e_{j},
$$

donde la variable dependiente $\mathrm{Yj}$ es el valor de desempeño de la empresa $\mathrm{j}$, medido como el promedio de siete ítems considerados en el desempeño de mercado y desempeño financiero. $\beta_{0}$ es la constante; $\beta_{1}$, el coeficiente de la estrategia de Robinson y Pearce; $\beta_{2}$, el coeficiente de capacidades gerenciales, por extracción de los componentes principales de los siete ítems analizados; $\beta_{3}$, los Activos de la empresa, variable de control; y finalmente, ej, el error o el residual del modelo propuesto.

\section{Resultados}

Primer paso: diferencias entre variables, prueba U de Mann-Whitney

En el Cuadro 1 se muestran los valores medios y la desviación estándar de los ítems contemplados en la categoría de las capacidades directivas, diferenciados por Cava y Rioja, así como la significancia del Test U de Mann-Whitney para muestras independientes.

Cuadro 1. Test de U Mann-Whitney U de las Capacidades directivas para Cava DOP y Rioja DOP

\begin{tabular}{|l|c|c|c|c|c|}
\hline Capacidades directivas & $\begin{array}{c}\text { Media } \\
\text { Cava }\end{array}$ & DS Cava & $\begin{array}{c}\text { Media } \\
\text { Rioja }\end{array}$ & $\begin{array}{c}\text { DS } \\
\text { Rioja }\end{array}$ & $\begin{array}{c}\text { Sig. Test } \\
\text { de U } \\
\text { Mann- } \\
\text { Whitne } \\
\text { y }\end{array}$ \\
\hline Competencias de gestión & 2.85 & 0.75 & 3.18 & 0.92 & 0.15 \\
\hline $\begin{array}{l}\text { Conocimientos y habilidades de los } \\
\text { empleados }\end{array}$ & 3.05 & 0.69 & 3.57 & 0.87 & 0.01 \\
\hline Clima laboral & 3.50 & 0.61 & 3.75 & 0.92 & 0.20 \\
\hline Eficiencia de la estructura organizativa & 2.90 & 0.72 & 3.30 & 0.90 & 0.09 \\
\hline Coordinación & 3.30 & 0.66 & 3.48 & 0.90 & 0.46 \\
\hline Planteamiento estratégico para atraer empleados & 2.35 & 0.88 & 2.91 & 0.94 & 0.03 \\
\hline $\begin{array}{l}\text { Habilidad } \\
\text { creativos }\end{array}$ & & 0.62 & 3.11 & 0.89 & 0.16 \\
\hline
\end{tabular}

Fuente: elaboración propia

El análisis del nivel de capacidades directivas revela un menor nivel de significación estadística en seis de los siete ítems analizados para la DOP Cava. Para el primero, con un nivel de significación <0,05, Cava está a la zaga de Rioja en las áreas de conocimientos y habilidades de los empleados, planificación estratégica y capacidad de atracción de empleados creativos. Para el segundo, con un nivel de significación entre 0,05 y 0,10, el Cava es superado por Rioja en el área de estructura organizativa eficiente, y para el tercero, con un nivel de significación entre 0,10 y 0,20, Cava sigue a Rioja en las áreas de competencias directivas, trabajo clima y planificación estratégica.

Segundo paso: regresión bayesiana 
En cuanto a la regresión bayesiana, (Cuadro 2) muestra el resultado de las cuatro estrategias genéricas de Robinson \& Pearce para las DOP de Cava y las capacidades directivas.

Cuadro 2. Regresión Bayesiana para capacidades directivas y las estrategias genéricas de Robinson y Pearce para DOP Cava. Variable dependiente el desempeño empresarial.

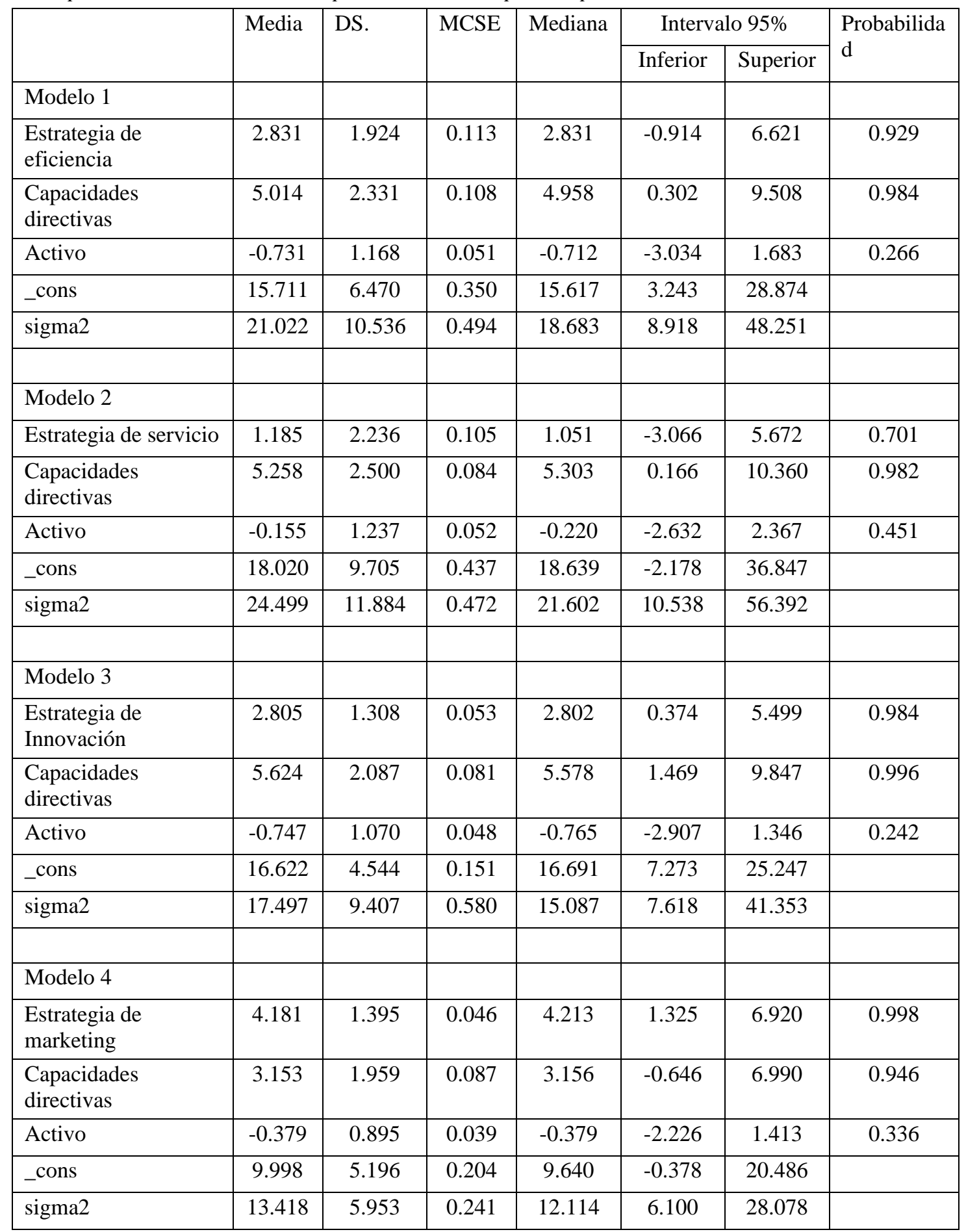

Fuente: elaboración propia

Los resultados de la regresión bayesiana efectuada, con las cuatro estrategias genéricas, las capacidades de gestión y el desempeño empresarial para la DOP Cava, muestran una elevada relación, en los cuatro modelos, entre capacidad gerencial y desempeño (entre 95 y 98\%). En cuanto a las estrategias, no existe una única estrategia que se relacione con el desempeño, pero dos de ellas muestran una importante asociación causa-efecto, la estrategia de marketing $(99,8 \%)$ y la estrategia de innovación $(98,4 \%)$. Finalmente, con respecto a las otras dos estrategias que se estudian en este trabajo, eficiencia (Porter: estrategia de costos) y servicio (Porter: estrategia de diferenciación), la estrategia de eficiencia (92,9\%), 
puede correlacionarse con el desempeño empresarial ya que su significancia estadística es menor a 0,10. Por el contrario, la estrategia de servicio (70\%) no se puede correlacionar con el desempeño empresarial.

\section{Conclusiones}

El estudio de las capacidades directivas en la DOP Cava y el desempeño empresarial, muestra como las capacidades directivas deben ser fomentadas en la DOP Cava por dos razones al menos, la primera por un nivel de dotación inferior a la DOP de referencia Rioja, y en segundo término por la alta relación que tienen con el mejor desempeño empresarial.

\section{Bibliografía}

Baruch, Y. y Holtom, BC. (2008). "Survey response rate levels and trends in organizational research". Human Relations 61(8):1139-1160.

Block, J. H., Jaskiewicz, P., y Miller, D. (2011). "Ownership versus management effects on performance in family and founder companies: A Bayesian reconciliation”. Journal of Family Business Strategy, 2(4), 232-245.

CRC, (2021). Cava - History. Consejo Regulador del Cava, https://www.cava.wine/es/el-consejoregulador-cava/la-denominacion-de-origen-cava/la-historia-del-cava (accessed 23 February 2021).

Fernández, E. y Pinilla, V. (2014). "Historia económica del vino en España (1850-2000)". In: La economía del vino en España y en el mundo; Castillo JS, Compés R (eds.). pp. 67-98. Cajamar Caja Rural, Almeria.

MAPA, (2020). Alimentacion. Calidad agroalimentaria, calidad diferenciada. Denominaciones de origen. Ministerio de Agricultura, Pesca y Alimentación, https://www.mapa.gob.es/es/alimentacion/temas/calidad-agroalimentaria/calidad-diferenciada/ (accessed 23 January 2021).

Robinson, RB. y Pearce, JA. (1988). "Planned patterns of strategic behavior and their relationship to business-unit performance". Strategic Management J 9(1): 43-60.

SABI, (2017). Sistema de Análisis de Balances Ibéricos, https://sabi.bvdinfo.com.

Saito, Y. y Takenaka, K. (2004). "Development of wine industry in Spain: three pioneer regions in commercial wine production". Geographical Review of Japan 77(5): 241-261. 